\title{
Step-down therapy in well-controlled asthmatic patients using salmeterol xinafoate/fluticasone propionate combination therapy
}

\section{Kazuya Horiuchi \\ Keita Kasahara \\ Yusuke Kuroda \\ Haruna Morohoshi \\ Yosuke Hagiwara \\ Gen Ishii}

Respiratory Disease Center, Showa University Northern Yokohama Hospital, Yokohama-shi, Kanagawa-ken, Japan
Correspondence: Kazuya Horiuchi Respiratory Disease Center, Showa University Northern Yokohama Hospital, 35-I Chigasaki-chuou,Tsuzuki-ku, Yokohama-shi, Kanagawa-ken 224-8503, Japan

Tel +8I 459497000

Fax +8I 459497117

Email poricelona0921@gmail.com
This article was published in the following Dove Press journal:

Journal of Asthma and Allergy

18 March 2016

Number of times this article has been viewed

Purpose: A combination therapy with inhaled corticosteroid (ICS) and a long-acting $\beta$ agonist (LABA) is the standard treatment for asthmatic patients, and step-down treatment is recommended once control has been achieved. However, little data exist that evaluate the long-term outcomes after step-down treatment.

Objective: To compare the long-term outcomes of step-down therapy with ICS/LABA or ICS alone for asthmatic patients who have achieved well-controlled asthma by the ICS $(250 \mu \mathrm{g}$ fluticasone)/LABA (50 $\mu \mathrm{g}$ salmeterol) combination (SFC, two puffs per day).

Patients and methods: We randomized 40 well-controlled patients with asthma receiving SFC $(250 \mu \mathrm{g})$ to two groups; one group of patients received step-down therapy with low-dose SFC (100 $\mu \mathrm{g}$, two puffs daily) and another group of patients received step-down therapy with high-dose fluticasone propionate (FP) alone (500 $\mu \mathrm{g}$, daily). The two groups were monitored over 12 months for changes in asthma control test scores, respiratory function (percent forced expiratory volume in 1 second, maximal expiratory flow rate at $50 \%$ of the vital capacity $\left[\% \mathrm{FEF}_{50}\right]$, and maximal expiratory flow rate at $25 \%$ of the vital capacity $\left[\% \mathrm{FEF}_{25}\right]$ ), and the concentration of fractional exhaled nitric oxide.

Results: There was no significant difference in the dropout rate between the SFC and FP groups. Low-dose SFC maintained the stability of all parameters over 12 months, whereas the FP group exhibited a rapid 5\% decrease in forced expiratory volume in 1 second within 2 months after discontinuation of salmeterol; furthermore, after 10 months, there was a gradual decrease in $\% \mathrm{FEF}_{50}$ and $\% \mathrm{FEF}_{25}$.

Conclusion: This study suggests that a balanced step-down protocol, including both ICS and LABA, is essential in providing long-term stability to patients with mild-to-moderate wellcontrolled asthma.

Keywords: long-term outcome, asthma, fluticasone propionate, salmeterol, step down

\section{Introduction}

Asthma is a chronic inflammatory airway disease characterized by marked eosinophilic inflammation, leading to progressive airway remodeling and impaired airflow. Therefore, asthma treatment includes the use of anti-inflammatory drugs to reduce the progression of airway impairment at an early stage. The inhaled corticosteroid (ICS) is a standard anti-inflammatory treatment for patients with asthma. Moreover, a combination therapy with ICS and long-acting $\beta$ agonists (LABAs) improves respiratory function and asthma symptoms and also reduces the frequency of usage of short-acting $\beta$ agonists in comparison with ICS alone. ${ }^{1,2}$ 
The Global Initiative for Asthma (GINA) guidelines recommend that once asthma control has been achieved and maintained for at least 3 months, step-down therapy can be attempted under careful monitoring. For ICS/LABA combination therapies, the guidelines suggest to reduce the dose of ICS while continuing LABA because few studies suggest that low-dose ICS with LABA is more effective in reducing the frequency of exacerbations than fixed doses of ICS without LABA. On the other hand, the US Food and Drug Administration recommends that LABA be discontinued once asthma control has been achieved or not be used when asthma control is achieved with low-to-medium doses of ICS for safety concerns. ${ }^{3,5}$ Meta-analyses on safety of LABA in asthma clinical trials suggest that LABA monotherapy may increase the risk of death related to asthma. ${ }^{4}$

The present randomized prospective study compared the long-term outcomes of step-down therapy with ICS/ LABA or ICS alone for asthmatic patients who had achieved well-controlled asthma levels with ICS (fluticasone)/LABA (salmeterol) combination therapy.

\section{Material and methods Study subjects}

Between January 2011 and January 2013, adult patients with mild-to-moderate persistent asthma were recruited at the Showa University Northern Hospital, Yokohama, Kanagawa, Japan. Asthma severity and control conditions were assessed according to the GINA 2012 guidelines. Based on the guidelines, patients with intermittent, mild moderate, or moderate persistent asthma were included in the study. Patients had achieved well-controlled asthma for at least 6 months using two puffs daily of salmeterol xinafoate $(50 \mu \mathrm{g})$ /fluticasone propionate $(\mathrm{FP} ; 250 \mu \mathrm{g})$ combination (SFC). Exclusion criteria included the use of other LABA drugs, symptoms of chronic obstructive pulmonary disease (COPD), and being a current smoker. We verified that the Brinkman indexes of enrolled ex-smokers were $<100$ for differentiating asthma and COPD in ex-smokers. Moreover, all of them underwent highresolution computed tomography scanning, and attending doctors and radiologist confirmed that they had no attenuation areas. Additionally, considering the reversibility of forced expiratory volume in 1 second $\left(\mathrm{FEV}_{1}\right)$ following a bronchodilator administration of $>12 \%$ at the initial examination, asthma was diagnosed in ex-smokers. The study was approved by the Showa University Northern Yokohama Hospital Ethics Committee, and all patients gave written informed consent. The Clinical Trial Registration Number is 1008-03.

\section{Study design}

During this prospective randomized study, patients were randomized into two groups using the envelope method: patients who received step-down therapy with SFC $100 \mu \mathrm{g}$ (two puffs daily) comprised the SFC group and patients who received step-down therapy with FP $500 \mu \mathrm{g}$ daily comprised the FP group. The use of histamine receptor blocker and leukotriene receptor antagonists was allowed as long as the prescription was not changed during this study. The maintenance of $>80 \%$ compliance was verified during regular visits. Over the 12-month follow-up period after initiation of the step-down therapy, patients who experienced exacerbation and required the use of short oral systemic corticosteroids or those who needed to modify the treatment due to poor asthma control were treated as dropouts and their data were excluded from analysis.

\section{Outcome parameters}

Patients were monitored every 2 months for changes in respiratory function, asthma control test (ACT) score, and the concentration of fractional exhaled nitric oxide (FeNO) before the initiation of the step-down therapeutic protocol (ie, at baseline) and over the following 12 months. Monitored respiratory functions included percent forced expiratory volume in 1 second $\left(\% \mathrm{FEV}_{1}\right)$, maximal expiratory flow rate at $50 \%$ of the vital capacity $\left(\% \mathrm{FEF}_{50}\right)$, and maximal expiratory flow rate at $25 \%$ of the vital capacity $\left(\% \mathrm{FEF}_{25}\right)$. In addition, the $\mathrm{FEV}_{1}$ absolute decline rate was compared between the groups at 12 months. The ACT test was used to verify that the patient maintained wellcontrolled asthma levels, characterized by an ACT score $\geq 20$, according to the GINA guidelines. ${ }^{6}$ FeNO was measured by a portable device, NObreath (Bedfont Scientific Ltd, Maidstone, UK). ${ }^{7}$

\section{Data analysis}

Data were collected before the initiation of the step-down therapeutic protocol (ie, at baseline [0 weeks]), as well as after 2 months, 4 months, 6 months, 8 months, 10 months, and 12 months. Next, comparisons between the SFC group and the FP group at specific time points were performed using the Mann-Whitney $U$ test. In this study, patients were randomly assigned; however, there was a significant difference in baseline $\% \mathrm{FEF}_{25}$ values between the SFC and $\mathrm{FP}$ groups. Therefore, we evaluated respiratory functions using relative changes over time and not absolute values. Likewise, FeNO data were expressed as relative changes over time. 


\section{Results}

Forty asthmatic patients met the inclusion/exclusion criteria and were randomly assigned to the SFC group $(n=20)$ or the FP group $(n=20)$. The two groups exhibited similar baseline characteristics, except for the elevated $\% \mathrm{FEF}_{25}$ of the SFC group $(P<0.001$; Table 1). Patients were predominantly adult males in the age range of 50-55 years. One-third of the patients in each group were ex-smokers with no signs or diagnosis of COPD according to exclusion criteria. The average ACT scores were consistent with well-controlled asthma, and lung functions were compatible with mild-to-moderate disease severity.

In the SFC group, two patients dropped out after initiation of step-down therapy. There were three drop-out patients in the FP group. In all cases, the cause of dropout was the exacerbation of asthma that assumed respiratory infection. The mean period until dropout was 8 months in the SFC group and 8.3 months in the FP group. There was no significant difference in the dropout rate of the SFC and FP groups (logrank sum test; Figure 1). Accordingly, the following analyses were performed on a total of 35 patients for the SFC group $(\mathrm{n}=17)$ and the FP group $(\mathrm{n}=18)$.

The two step-down therapeutic protocols did not affect ACT scores, which remained relatively stable over the 12-month follow-up period (Figure 2). Likewise, there was no significant change in FeNO levels before and after the initiation of the step-down therapeutic protocols between the two groups (Figure 3). Collectively, these data suggest that

Table I Characteristics of enrolled patients

\begin{tabular}{|c|c|c|c|}
\hline & $\begin{array}{l}\text { FP } \\
\text { group }\end{array}$ & $\begin{array}{l}\text { SFC } \\
\text { group }\end{array}$ & $\begin{array}{l}\text { Mann-Whitney } \\
U \text { test }\end{array}$ \\
\hline$N$ & 20 & 20 & \\
\hline Age (years old) & $55.8 \pm 9.6$ & $49.9 \pm 12.9$ & NS \\
\hline Sex (male/female) & $8 / 12$ & $9 / 11$ & \\
\hline \multicolumn{4}{|l|}{ Other drugs } \\
\hline $\mathrm{HI}$ receptor antagonists & 5 & I & \\
\hline $\begin{array}{l}\text { Leukotriene receptor } \\
\text { antagonists }\end{array}$ & 0 & 2 & \\
\hline ACT score & $24.7 \pm 0.7$ & $24.9 \pm 0.3$ & NS \\
\hline FeNO (ppb) & $29.0 \pm 21.5$ & $32.6 \pm 23.8$ & NS \\
\hline $\mathrm{FEV}_{\mathrm{I}} / \mathrm{FVC}(\%)$ & $72.8 \pm 10.1$ & $78.7 \pm 10.4$ & NS \\
\hline$\% \mathrm{FEV}_{1}$ & $93.1 \pm 10.9$ & $94.0 \pm 12.0$ & NS \\
\hline$\% \mathrm{FEF}_{50}$ & $46.2 \pm 17.7$ & $69.6 \pm 36.0$ & NS \\
\hline$\% \mathrm{FEF}_{25}$ & $26.8 \pm 14.9$ & $56.7 \pm 33.4$ & $P<0.00$ I \\
\hline Bronchodilator response (\%) & 16.0 & 17.9 & \\
\hline
\end{tabular}

Note: Data are presented as mean \pm standard deviation.

Abbreviations: FP, fluticasone propionate; SFC, salmeterol xinafoate/fluticasone propionate combination; ACT, asthma control test; NS, not significant; FeNO, fractional exhaled nitric oxide; $\mathrm{FEV}_{1}$, forced expiratory volume in I second; \%FEV percent forced expiratory volume in I second; $\% \mathrm{FEF}_{50}$, maximal expiratory flow rate at $50 \%$ of the vital capacity; $\% \mathrm{FEF}_{25}$, maximal expiratory flow rate at $25 \%$ of the vital capacity.

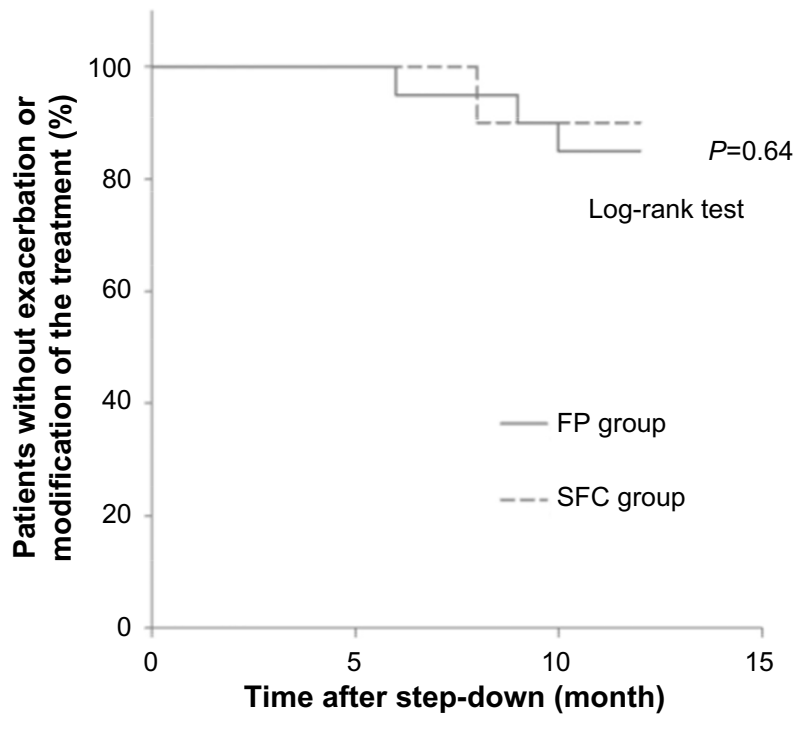

Figure I Clinical time courses of patients after step-down. Kaplan-Meier plot of the percentage of patients without exacerbations or modification of the treatment Abbreviations: FP, fluticasone propionate; SFC, inhaled corticosteroid/long-acting $\beta$ agonist combination.

bronchial inflammation was equally controlled by low-dose ICS in the SFC group and high-dose ICS in the FP group.

Regarding lung function, $\% \mathrm{FEV}_{1}$ was not affected by the SFC step-down therapeutic protocol (Figure 4). In contrast, $\% \mathrm{FEV}_{1}$ significantly decreased within the first 2 months after the initiation of the FP step-down therapeutic protocol and then remained stable during the remaining follow-up period. Accordingly, there was a significant difference in $\% \mathrm{FEV}_{1}$

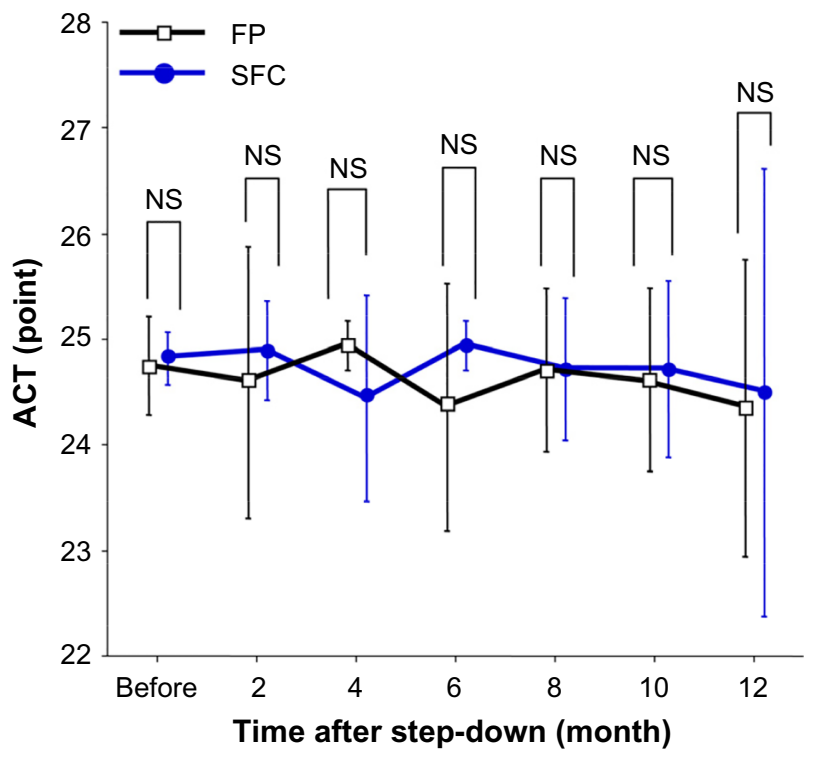

Figure 2 Time course changes in the scores of ACT during the study. Abbreviations: FP, fluticasone propionate; SFC, inhaled corticosteroid/long-acting $\beta$ agonist combination; ACT, asthma control test; NS, not significant. 


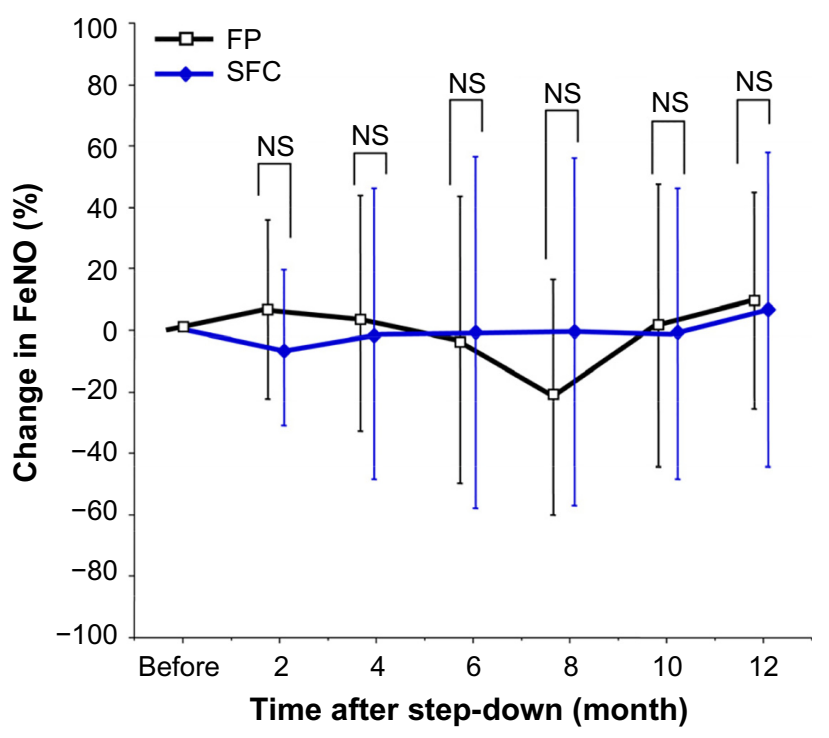

Figure 3 Time course changes in the values of FeNO during the study. Abbreviations: FP, fluticasone propionate; SFC, inhaled corticosteroid/long-acting $\beta$ agonist combination; FeNO, fractional exhaled nitric oxide; NS, not significant.

between the FP and SFC groups over the entire 2- to 12-month period $(P<0.02)$.

The $\mathrm{FEV}_{1}$ absolute decline rate of the SFC group was $-18.0 \mathrm{~mL} /$ year, whereas that of the FP group was $-144 \mathrm{~mL} /$ year (Figure 5). The FEV 1 decline rate of the SFC group was significantly slower than that of the FP group as is the case with the relative changes in $\% \mathrm{FEV}_{1}(P<0.05)$.

The SFC step-down therapeutic protocol did not significantly affect $\% \mathrm{FEF}_{50}$ over the entire 12 -month follow-up period (Figure 6). In contrast, the FP step-down

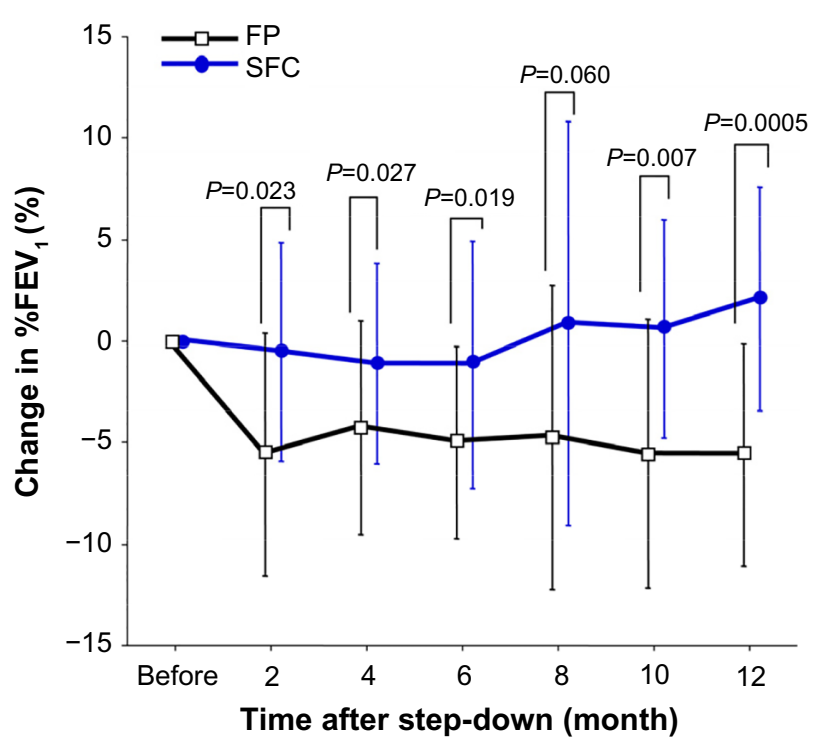

Figure 4 Time course changes in \%FEV, during the study.

Abbreviations: FP, fluticasone propionate; SFC, inhaled corticosteroid/long-acting $\beta$ agonist combination; \%FEV 1 , percent forced expiratory volume in I second.

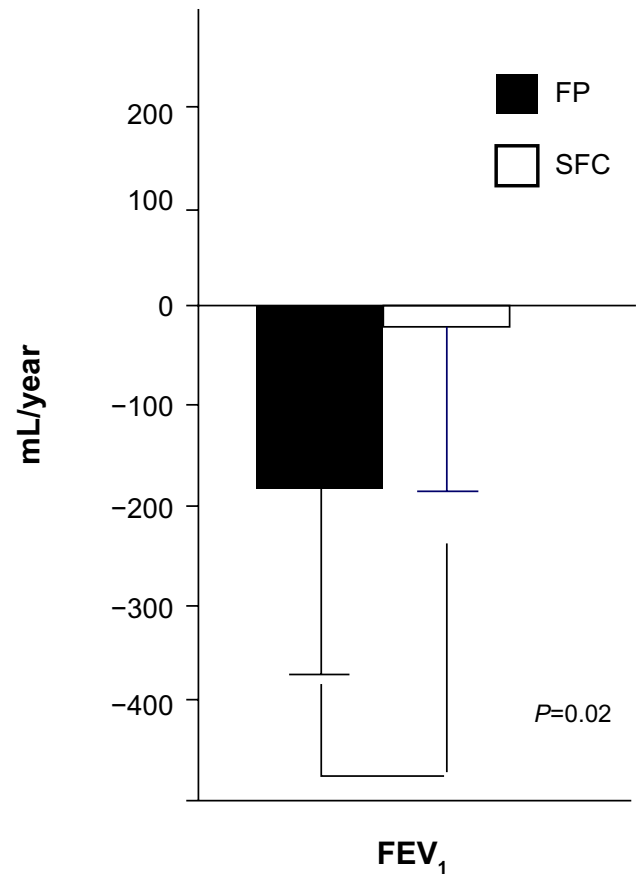

Figure $5 \mathrm{FEV}$, decline rate over I year.

Abbreviations: FP, fluticasone propionate; SFC, inhaled corticosteroid/long-acting $\beta$ agonist combination; $\mathrm{FEV}_{1}$, forced expiratory volume in I second.

therapeutic protocol induced a progressive decrease in $\% \mathrm{FEF}_{50}$ values, which became significantly different from those of the SFC group during the 8-12-month period $(P<0.03)$. On the other hand, the two step-down therapeutic protocols did not affect $\% \mathrm{FEF}_{25}$ values, except for significantly lower values in the FP group than those in

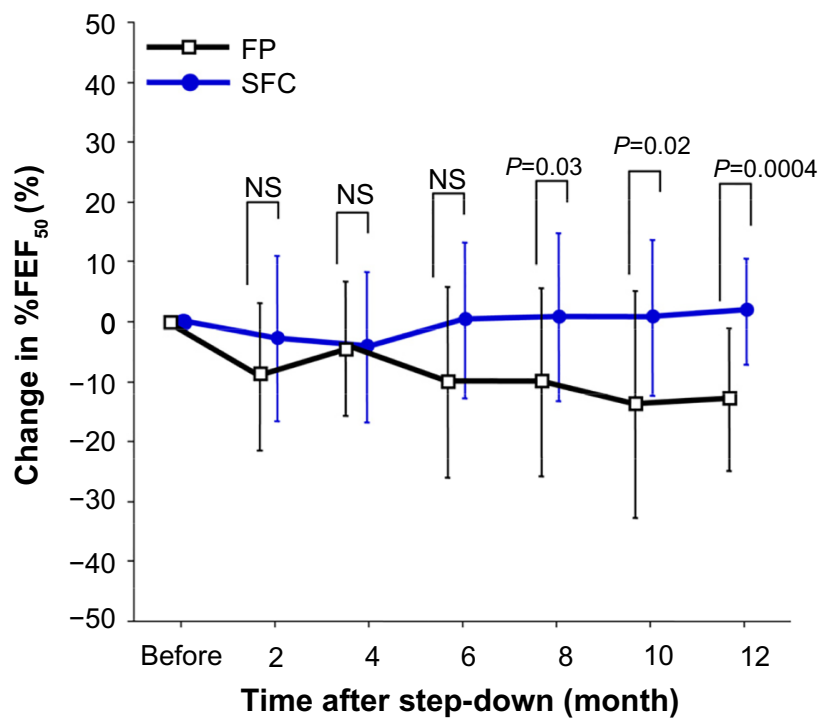

Figure 6 Time course changes $\% \mathrm{FEF}_{50}$ during the study.

Abbreviations: FP, fluticasone propionate; SFC, inhaled corticosteroid/long-acting $\beta$ agonist combination; NS, not significant; $\% \mathrm{FEF}_{50}$, maximal expiratory flow rate at $50 \%$ of the vital capacity. 


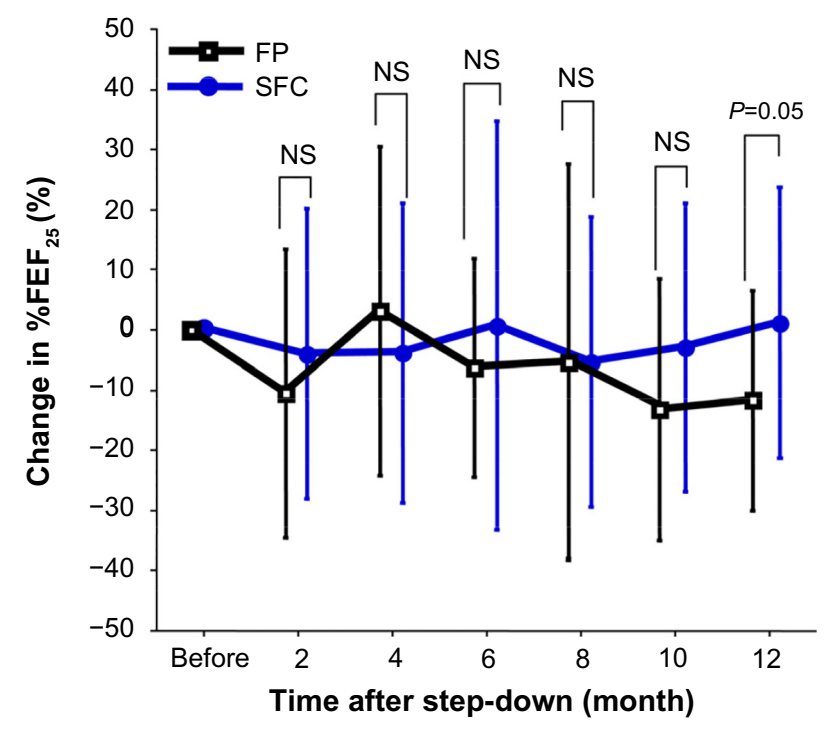

Figure 7 Time course changes in $\% \mathrm{FEF}_{25}$ during the study.

Abbreviations: FP, fluticasone propionate; SFC, inhaled corticosteroid/long-acting $\beta$ agonist combination; NS, not significant; $\% \mathrm{FEF}_{25}$, maximal expiratory flow rate at $25 \%$ of the vital capacity.

the SFC group at the 12-month visit $(P<0.05$; Figure 7). These data suggest that both $\% \mathrm{FEF}_{50}$ and $\% \mathrm{FEF}_{25}$ respond to LABA discontinuation by a gradual decrease over time; however, $\% \mathrm{FEF}_{50}$ represents a more sensitive marker of treatment response.

\section{Discussion}

This study is based on the current GINA guidelines that recommend step-down therapy once asthma control has been achieved and maintained for 3-6 months. Meta-analyses suggest that reducing the ICS dose while maintaining LABA is more likely to maintain asthma under control and to reduce the rate of severe exacerbation than discontinuing LABA while maintaining the ICS dose for several months. ${ }^{8-11}$ However, a few studies have reported the adverse effects of long-term LABA inhalation, which may worsen airway inflammation and increase the risk of death related to exacerbation, ${ }^{4,12}$ which is in agreement with US Food and Drug Administration's warnings. These studies suggest that the most appropriate step-down therapeutic protocol would be to reduce both ICS and LABA in tandem, instead of reducing or eliminating only one of them. Accordingly, the present study compared the long-term outcomes of a balanced step-down therapeutic protocol (from SFC $250 \mu \mathrm{g}$ to SFC $100 \mu \mathrm{g}$, two puffs each day) and an ICS-only protocol (FP $500 \mu \mathrm{g}$ daily).

The patients were monitored for changes in ACT scores, FeNO levels, and respiratory function over a 12-month period. The first parameters remained stable during the 12-month follow-up, regardless of the step-down period. In contrast, the
$\% \mathrm{FEV}_{1}$ of the $\mathrm{FP}$ group decreased significantly immediately after discontinuing LABA. This result suggests that LABA is effective in improving lung functions in mild-to-moderate persistent asthmatic patients, despite their conditions being well controlled for at least 6 months as reported in other studies. ${ }^{1,2}$ On the other hand, FeNO levels did not increase in the SFC group despite ICS dose reduction. In addition, there were no significant differences between the two groups in ACT and dropout rate over the entire year. It appears that LABA is also effective in terms of improving respiratory functions in patients with mildly or moderately persistent asthma, and low-dose ICS may be enough for such patients to relieve their inflammations even if they continue LABA for a long period. On the other hand, the Formoterol and Corticosteroids Establishing Therapy study reported that in patients with severe asthma (not mild or moderate asthma as in this study), the exacerbation rate is increased significantly in those who were treated with low-dose ICS and LABA combination therapy compared with those who were treated with high-dose ICS alone. ${ }^{2}$ It appeared that low-dose ICS was not enough for severe asthma, and it became the cause of potential revival of bronchial inflammation. This suggests that it is important to consider how to step down the treatment while evaluating the inflammatory conditions of asthma.

The impact of the step-down therapeutic protocol on the status of the peripheral bronchi was assessed by the measurements of forced expiratory flow rates. The standard $250 \mathrm{mg}$ SFC treatment is known to improve $\% \mathrm{FEF}_{25}$ and $\% \mathrm{FEF}_{50}$ in asthmatic patients. The present study shows that stepping down from $250 \mathrm{mg}$ to $100 \mathrm{mg}$ SFC did not significantly affect $\% \mathrm{FEF}_{25}$ or $\% \mathrm{FEF}_{50}$ values of the asthmatic patients, suggesting that their asthma remained under control for at least 12 months. In contrast, the absence of LABA in the FP step-down therapeutic protocol caused a gradual decrease in $\% \mathrm{FEF}_{50}$, reaching $90 \%$ of baseline values after 12 months. These data suggest that the continuous use of low-dose LABA with low-dose ICS may prevent the progression of peripheral airway impairment. It has been reported that airway remodeling progresses only for airway contraction even if respiratory tract inflammation is controlled. ${ }^{13-15}$ In the SFC group, airway inflammation was relieved to the same extent as in the FP group, and the continuous use of LABA may prevent airway contraction. As a result, impaired airflow of the peripheral airways was not exacerbated in the SFC group.

\section{Conclusion}

In conclusion, this study demonstrates that in patients with mild-to-moderate asthma, well controlled with $250 \mathrm{mg} \mathrm{SFC}$, 
a balanced step-down therapeutic protocol with $100 \mathrm{mg}$ SFC efficiently maintains the FeNO level, ACT score, and $\% \mathrm{FEV}_{1}$, $\% \mathrm{FEF}_{25}$, and $\% \mathrm{FEF}_{50}$ values stable for at least 12 months. In contrast, switching to high-dose ICS alone led to a rapid increase in airflow impairment in the upper respiratory tract and a gradual deterioration of peripheral airflow over 12 months. These data suggest that step-down therapeutic protocols should include low-dose LABA to prevent the progression of airflow impairment and improve the long-term prognosis of asthmatic patients under step-down therapy.

\section{Acknowledgment}

The authors would like to thank Enago (www.enago.jp) for the English language review.

\section{Disclosure}

The authors have no conflicts of interest in this work.

\section{References}

1. Greening AP, Ind PW, Northfield M. Shaw G. Added salmeterol versus higher-dose corticosteroid in asthma patients with symptoms on existing inhaled corticosteroid. Allen and Hanburys Limited UK Study Group. Lancet. 1994;344(8917):219-224.

2. Pauwels RA, Löfdahl CG, Postma DS, et al. Effect of inhaled formoterol and budesonide on exacerbation of asthma: formoterol and corticosteroids establishing therapy (FACET) International Study Group. N Eng J Med. 1997;337(20):1405-1411.

3. Kramer JM. Balancing the benefits and risks of inhaled long-acting beta-agonists the influence of values. $N$ Engl J Med. 2009;360(16): 1592-1595.

4. Nelson HS, Weiss ST, Bleecker ER, et al; SMART Study Group. The salmeterol multicenter asthma research trial: a comparison of usual pharmacotherapy for asthma or usual pharmacotherapy plus salmeterol. Chest. 2006;129(1):15-26.
5. Chowdhury BA, Dal Pan G. The FDA and safe use of long-acting beta-agonists in the treatment of asthma. N Engl J Med. 2010;362(13): 1169-1171.

6. Thomas M, Kay S, Pike J, et al. The asthma control test (ACT) as a predictor of GINA guideline-defined asthma control: analysis of a multinational cross-sectional survey. Prim Care Respir J. 2009;18(1):41-49.

7. Saito J, Gibeon D, Macedo P, Menzies-Gow A, Bhavsar PK, Chung KF. Domiciliary diurnal variation of exhaled nitric oxide fraction for asthma control. Eur Respir J. 2014;43(2):474-484.

8. Godard P, Greillier P, Pigearias B, Nachbaur G, Desfougeres JL, Attali V. Maintaining asthma control in persistent asthma: comparison of three strategies in a 6-month double blind randomised study. Respir Med. 2008;102(8):1124-1131.

9. Brozek JL, Kraft M, Krishnan JA, et al. Long-acting $\beta 2$-agonist step-off in patients with controlled asthma. Arch Intern Med. 2012;172(18):1365-1375.

10. Wells KE, Peterson EL, Ahmedani BK, Severson RK, GleasonComstock J, Williams LK. The relationship between combination inhaled corticosteroid and long-acting $\beta$-agonist use and severe asthma exacerbations in a diverse population. J Allergy Clin Immunol. 2012;129(5):1274-1279.

11. Pedersen S, O'Byrne PM. Combination inhaled corticosteroid and long-acting $\beta 2$-agonist use and severe asthma exacerbations. $J$ Allegy Clin Immunol. 2012;129(5):1280-1281.

12. Salpeter SR, Wall AJ, Buckley NS. Long-acting beta-agonists with and without inhaled corticosteroids and catastrophic asthma events. Am J Med. 2010;123(4):322-328.

13. Adachi M, Aizawa H, Ishihara K, et al. Comparison of salmeterol/ fluticasone propionate (FP) combination with FP + sustained release theophylline in moderate asthma patients. Respir Med. 2008;102(7):1055-1064.

14. Ketai L, Harkins M, Fiato KL, Iwamoto GK. Exhaled nitric oxide and bronchial wall thickening in asthmatics during and after acute exacerbation: evidence of bronchial wall remodeling. $J$ Asthma. 2005;42(8):667-671.

15. Grainge CL, Lau LC, Ward JA, et al. Effect of bronchoconstriction on airway remodeling in asthma. N Eng J Med. 2011;364(21):2006-2015.
Journal of Asthma and Allergy

\section{Publish your work in this journal}

The Journal of Asthma and Allergy is an international, peer-reviewed open-access journal publishing original research, reports, editorials and commentaries on the following topics: Asthma; Pulmonary physiology; Asthma related clinical health; Clinical immunology and the immunological basis of disease; Pharmacological interventions and

\section{Dovepress}

new therapies. Issues of patient safety and quality of care will also be considered. The manuscript management system is completely online and includes a very quick and fair peer-review system, which is all easy to use. Visit http://www.dovepress.com/testimonials.php to read real quotes from published authors. 